\title{
Quetiapine versus risperidone in the pharmacotherapy of behavioral and psychological symptoms of dementia: an open labelled study
}

\begin{abstract}
Dementia syndrome consists of behavioral and psychological symptoms (BPSD) such as non-cognitive disturbances including affective symptoms like anxiety, depression, irritability, psychotic symptoms, and behavioral disorders like insomnia, wandering, aggression, agitation, eating and sexual disorders. Since there are no parallel studies of two atypical antipsychotics in the treatment of BPSD in India, the present study is head-to- head comparison of efficacy and tolerability of Quetiapine and risperidone in the treatment of patients of dementia with BPSD.

Methodology: 30 patients received oral flexible dose of Quetiapine $50-400 \mathrm{mg} /$ day once a day and 30 patients in the other arm received oral flexible dose of Risperidone $0.5-6 \mathrm{mg} /$ day once a day for two months. Patients were followed up once a month, assessment was done at the end of 8 weeks of treatment. Efficacy of the drug was assessed by using Neuro psychiatry inventory scale [NPI] and Clinical global impression scale [CGI] abnormal involuntary movement scale [AIIMS] compared between the two arms to assess the clinical cure at the end of the study.

Results: EPS score of $>12$ was seen in $61.2 \%$ of the people who received Risperidone and $38 \%$ who received Quetiapine ( $\mathrm{p}$ value: 0.022 ). Risperidone was found to be more cardio safe, efficacious for aggression, psychotic symptoms than Quetiapine in 57.1\% of the study population.

Conclusions: The results of this study suggest that Quetiapine is as effective as risperidone in treating psychotic symptoms. It has comparable over all tolerability, may have a more favorable EPS profile. Patients are not at a higher propensity to develop cardiac complications with Quetiapine.
\end{abstract}

Keywords: Quetiapine, risperidone, neuro psychiatry, patients, psychotic symptoms
Volume 7 Issue 5 - 2019

Vyjayanthi Subramanian

MS Ramaiah Medical College, RGUHS University, India

Correspondence: Vyjayanthi Subramanian, MS Ramaiah Medical College, RGUHS University, Karnataka, India, Tel + 91 948I474768, Email vyjayanthi23@gmail.com

Received: July 1 I, 2019 | Published: October 21, 2019
Abbreviations: BPSD, behavioral and psychological symptoms of dementia; NPI, neuro psychiatry inventory scale; CGI, clinical global impression scale; AIIMS, abnormal involuntary movement scale; EPS, extrapyramidal symptoms; OPD, out-patient department

\section{Introduction}

Dementia syndrome is characterized by a progressive deterioration of cortical functioning including language, judgment, comprehension, memory, thinking and new learning. ${ }^{1}$ Dementia is common among patients with neurodegenerative disorders and substance abuse. ${ }^{2}$ In Asia pacific region the prevalence of dementia in 2015 is 23 million. ${ }^{1}$ Dementia syndrome consists of behavioral and psychological symptoms (BPSD) such as non-cognitive disturbances including affective symptoms like anxiety, depression, irritability, psychotic symptoms like delusions, hallucinations ,behavioral disorders like insomnia, wandering, aggression, agitation, eating and sexual disorders.

Behavioral and psychological symptoms affect $75 \%$ of the patients with dementia and are a major contributing factor responsible for distress to caregivers and hospitalization of patients. ${ }^{4}$ Treatment options for BPSD are typical and atypical antipsychotics. Because of profound adverse effects profile of conventional antipsychotics made them to be withdrawn from the treatment of BPSD. ${ }^{3}$ Enhanced efficacy and reduced extra pyramidal adverse effect profile of atypical antipsychotics have replaced unsatisfactory treatment with conventional antipsychotics for decades. ${ }^{5}$ There are studies describing the efficacy and safety of most frequently prescribed drugs Risperidone, Quetiapine and Olanzapine for dementia., ${ }^{4,6}$ Though both of the drugs are found to be efficacious and well tolerated in different studies, However, not many studies have aimed at showing the efficacy of antipsychotics in treating psychotic symptoms, since some of these delusions are related to cognitive dysfunction in which antipsychotics play a minimal role and tolerability of two most frequently prescribed atypical antipsychotics namely Risperidone and Quetiapine in the treatment of BPSD in India. It is thus considered desirable to take up the study to compare their efficacy and document the side effects of these two drugs.

\section{Background}

The pharmacodynamics action of newer atypical antipsychotics 
like Risperidone and Quetiapine are attributed to their action on both serotonergic and dopaminergic systems and offer a distinct advantage of decreased extra pyramidal symptoms and improved efficacy in patients with dementia. ${ }^{7,8}$ An open label ,flexible dose study showed an efficacy of Quetiapine and risperidone in the treatment of BPSD are $67.6 \%$ and $71 \%$ of clinical improvement with Clinical Global Impression scores and at low doses both of the drug are equally effective and generally well tolerated (including no cognitive impairment). ${ }^{3}$ Previous studies on the treatment of psychotic disorders showed that Quetiapine is as effective as Risperidone in the therapy of psychotic symptoms and is more effective for depressive symptoms, may have a more favorable EPS (extrapyramidal symptoms) profile, and has comparable overall tolerability. ${ }^{6}$ Since there are no parallel studies of two atypical antipsychotics in the treatment of BPSD in India, the present study is head-to- head comparison of efficacy and tolerability of Quetiapine and risperidone in the treatment outpatients of dementia with BPSD among the Indian population.

\section{Methodology}

To assess and compare the efficacy of oral Quetiapine versus oral Risperidone in the treatment of behavioral and psychological symptoms of dementia. To evaluate the tolerability of oral Quetiapine versus Risperidone in the treatment of behavioral and psychological symptoms of dementia.

\section{Materials and methods}

\section{Source of data}

The data will be collected from the patients visiting the Out-patient Department [OPD] of Psychiatry, Geriatric, Neurology M.S. Ramaiah Medical college and Hospitals.

\section{Methods of collection of data}

This will be an 8 weeks prospective comparative non-randomized, open label, flexible dose clinical study between the oral Quetiapine versus Risperidone on the efficacy and tolerability profile. All patients attending the Psychiatry, Geriatric, Neurology, and Medicine OPD will be screened for behavioral and psychological symptoms of dementia. Clinical diagnosis will be made by the treating consultants on the basis of DSM-4 Criteria ++and MMSE scale. 60 such patients diagnosed with behavioral and psychological symptoms of dementia will be recruited based on the inclusion and exclusion criteria to either of the two study groups. Demographic data with respect to age, gender, associated disease, habits, family history and drug history will be collected and a baseline serum prolactin and ECG will be done before starting the treatment. 30 patients received oral flexible dose of Quetiapine $50-400 \mathrm{mg} /$ day once a day and 30 patients in the other arm received oral flexible dose of Risperidone $0.5-6 \mathrm{mg} /$ day once a day for two months.

Patients recruited were followed up once a month in the psychiatry OPD assessment was done at the end of 8 weeks of treatment. If the patient complained of side effects at the end of one month of follow up dose of the drug was adjusted. Efficacy of the drug was assessed by using Neuro psychiatry inventory scale [NPI] and Clinical global impression scale [CGI]. Tolerability of the drug was assessed by abnormal involuntary movement scale [AIIMS] compared between the two arms to assess the clinical cure at the end of the study. Safety of the drug was measured by assessing the serum prolactin and ECG at the baseline and end of study.

\section{Inclusion criteria}

a. Patients in the age range of 40-85 years,

b. Patients with behavioral and psychological symptoms associated with dementia diagnosed by DSM - 4 criteria such as dementia due to neurodegenerative disorder, vascular dementia, and dementia due to alcohol abuse. ${ }^{6}$

c. Patients with MMSE score 10 to 26 ,

d. Patients willing to give a written informed consent for the study.

\section{Exclusion criteria}

a) Patients who are known or suspected to have hypersensitivity to Quetiapine or Risperidone.

b) Patients who have unstable medical or any clinically significant disease such as uncontrolled seizure disorder, active peptic ulcer, severe or unstable cardiovascular disease, acute or severe asthma condition, clinically significant abnormality in ECG and known liver disease. ${ }^{3}$

c) Patient on concurrent therapy with drugs having a possible drug reactions with risperidone/quetiapinelike:celecoxib, amidarone, cimetidine, fluoxetine, paroxetine, ketoconazole, erythromycin, diltiazem, ciprofloxacin, phenytoin.

d) Patients with raised prolactin level.

e) Pregnant and lactating mothers.

f) Patients who are currently on treatment with other atypical antipsychotics for last 4 months. ${ }^{3}$

g) Patients developing dementia after traumatic brain injury or sudden onset of dementia following cerebrovascular accident. ${ }^{3}$

\section{Sample size calculation}

Based on previous study conducted by Walter G. Deberdt.., Maurice W. Dysken: it was found that the NPI-Total score in Olanzapine group was $32.1+/-2$ and in Risperidone group was 30.6+/$0.2 .{ }^{4}$ In the present study, considering an effect size of 1.18 and alpha error of $5 \%$ and with the power of $90 \%$ sample size estimated was 25 in each group. However, considering lost to follow up of $20 \%$, it was decided to include 30 patients in each group. So sample is estimated to be 60 for this study.

\section{Statistical analysis}

All the quantitative variables like NPI scores, CGI score etc. were summarized using descriptive statistics such as mean and standard deviation or median and range. All the qualitative parameter like adverse events, type of dementia, gender etc. would be presented using frequency and percentages. Independent sample ' $t$ ' test / Mann Whitney U test was used to compare the difference in the mean NPI values between the Quetiapine and Risperidone. Paired ' $t$ ' test / Wilcox son's signed rank test was used to compare the difference in the NPI total scores and CGI scores from baseline to endpoint of study. Chi square test was used to compare the difference in proportion of categorical variables.

\section{Results}

In terms of tolerability, EPS score of $>12$ were seen in $61.2 \%$ of the people who received Risperidone and $38 \%$ who received 
Quetiapine ( $\mathrm{p}$ value: 0.022 ). Dry mouth was more in the group that received Risperidone ( $\mathrm{p}$ value: 0.027). At therapeutic doses, Risperidone was found to be more cardio safe. Baseline parameters such as height, weight, BMI were comparable in both the arms. For severity of aggression, Risperidone was found to be more efficacious than Quetiapine in $57.1 \%$ of the study population ( $\mathrm{p}$ value: 0.012 ). For treating delusions, Risperidone again was found to be beneficial (p value: $<0.001)$

\section{Discussion}

Few published studies provide direct comparisons of the atypical antipsychotic agents. The results of this study provide the first direct comparative data on quetiapine and risperidone in patients with behavioral and psychotic symptoms. Outpatient population was chosen to represent the patients most likely to be encountered by clinicians in community practice. Previous findings have demonstrated that EPS can lead to noncompliance. Therefore, greater tendency of treatment with risperidone to produce substantial EPS, compared with Quetiapine, could have an effect on patient compliance.

\section{Conclusions}

The results of this study suggest that Quetiapine is as effective as risperidone in treating psychotic symptoms.

It has comparable over all tolerability, may have a more favorable EPS profile. Care givers as well as the patients were more concerned with the EPS than sedation. In clinical practice this results in a bias to choose Quetiapine over Risperidone although the latter is efficacious in few parameters. Patients are not at a higher propensity to develop cardiac complications with Quetiapine.

\section{Conflicts of interest}

Author declares that there is no conflict of interest.

\section{References}

1. Chapter 1: Executive Summary. In: Dementia in Asia Pacific Region. London: Alzheimer's disease International; 2014. 4 p.

2. Kar N. Behavioral and psychological symptoms of dementia and their management. Indian J Psychiatry. 2009;51(Supp11):S77-S86.

3. Rainer M, Haushofer M, pfolz H, et al. Quetiapine versus risperidone in elderly patients with behavioral and psychological symptoms of dementia: Efficacy, safety and cognitive function. Eur Psychiatry. 2007;22(6):395-403

4. Deberdt W. Comparison of Olanzapine and Risperidone in the Treatmen of Psychosis and Associated Behavioral Disturbances in Patients with Dementia. Am J Geriatr Psychiatry. 2005;13(8):722-730.

5. Liperoti R, Pedone C, Corsonello A. Antipsychotics for the Treatment of Behavioral and Psychological Symptoms of Dementia (BPSD). Curr Neuropharmacol. 2008;6(2):117-124.

6. Mullen J, Jibson M, Sweitzer D. A comparison of the relative safety, efficacy, and tolerability of quetiapine and risperidone in outpatients with schizophrenia and other psychotic disorders: the quetiapine experience with safety and tolerability (QUEST) study. Clin Ther. 2001;23(11):1839-1854.

7. Motsinger C, Perron G, Lacy T et al. Use of Atypical Antipsychotic Drugs in Patients with Dementia. Am Fam Physician. 2003;67(11):2335-2340.

8. Rizzi L, Rosset I, Roriz-Cruz M. Global Epidemiology of Dementia: Alzheimer's and Vascular Types. BioMed Research International. $2014 ; 2014: 1-8$

\section{Acknowledgments}

None. 